\title{
Gallbladder Infection, CTCAE
}

National Cancer Institute

\section{Source}

National Cancer Institute. Gallbladder Infection, CT CAE. NCI Thesaurus. Code C143497.

A disorder characterized by an infectious process involving the gallbladder. 